This item was submitted to Loughborough's Research Repository by the author.

Items in Figshare are protected by copyright, with all rights reserved, unless otherwise indicated.

\title{
PGA-Net: pyramid feature fusion and global context attention network for automated surface defect detection
}

PLEASE CITE THE PUBLISHED VERSION

https://doi.org/10.1109/tii.2019.2958826

\section{PUBLISHER}

Institute of Electrical and Electronics Engineers (IEEE)

\section{VERSION}

AM (Accepted Manuscript)

\section{PUBLISHER STATEMENT}

(c) 2019 IEEE. Personal use of this material is permitted. Permission from IEEE must be obtained for all other uses, in any current or future media, including reprinting/republishing this material for advertising or promotional purposes, creating new collective works, for resale or redistribution to servers or lists, or reuse of any copyrighted component of this work in other works.

\section{LICENCE}

\section{All Rights Reserved}

\section{REPOSITORY RECORD}

Dong, Hongwen, Kechen Song, Yu He, Jing Xu, Yunhui Yan, and Qinggang Meng. 2019. "Pga-net: Pyramid Feature Fusion and Global Context Attention Network for Automated Surface Defect Detection".

Loughborough University. https://hdl.handle.net/2134/12249758.v1. 


\title{
PGA-Net: Pyramid Feature Fusion and Global Context Attention Network for Automated Surface Defect Detection
}

\author{
Hongwen Dong, Kechen Song, Yu He, Jing Xu, Yunhui Yan, Qinggang Meng, Senior Member, IEEE
}

\begin{abstract}
Surface defect detection is a critical task in industrial production process. Nowadays, there are lots of detection methods based on computer vision and have been successfully applied in industry, they also achieved good results. However, achieving full automation of surface defect detection remains a challenge, due to the complexity of surface defect, in intra-class, while the defects between inter-class contain similar parts, there are large differences in appearance of the defects. To address these issues, this paper proposes a pyramid feature fusion and global context attention network for pixel-wise detection of surface defect, called PGA-Net. In the framework, the multi-scale features are extracted at first from backbone network. Then the pyramid feature fusion module is used to fuse these features into five resolutions through some efficient dense skip connections. Finally, the global context attention module is applied to the fusion feature maps of adjacent resolution, which allows effective information propagate from low-resolution fusion feature maps to high-resolution fusion ones. In addition, the boundary refinement block is added to the framework to refine the boundary of defect and improve the result of predict. The final prediction is the fusion of the five resolutions fusion feature maps. The results of evaluation on four real-world defect datasets demonstrate that the proposed method outperforms the state-of-the-art methods on mean Intersection of Union and mean Pixel Accuracy (NEU-Seg: 82.15\%, DAGM 2007:

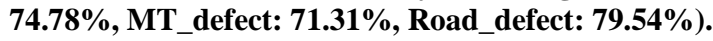

Index Terms - Surface defect detection, deep learning, deeplysupervised, pyramid feature fusion, global context attention, boundary refinement

\section{INTRODUCTION}

$\mathrm{T}$ HE quality is an important component during the manufacturing process. To meet the growing demand, it is necessary to ensure the quality of products strictly while improving the production efficiency in the process of industrial production. To meet the growing demand, it is necessary to ensure the quality of products strictly while improving the production efficiency in the process of industrial production.

This work is supported by the National Natural Science Foundation of China (51805078, 51374063), the National Key Research and Development Program of China (2017YFB0304200), the Fundamental Research Funds for the Central Universities (N170304014). (Corresponding authors: Kechen Song; Yunhui Yan)

H. Dong, K. Song, and Y. Yan are with the School of Mechanical Engineering and Automation, Northeastern University, Shenyang, Liaoning, 110819, China, and the Key Laboratory of Vibration and Control of AeroPropulsion Systems Ministry of Education of China, Northeastern University, Shenyang, 110819, China. (e-mail: donghongwenliran@163.com, songkc@me.neu.edu.cn, yanyh@mail.neu.edu.cn).

Q. Meng is with the department of computer science, Loughborough University, Loughborough LE11 3TU, U.K. (e-mail: q.meng@lboro.ac.uk).

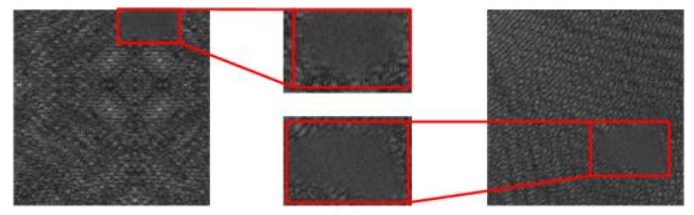

(a)

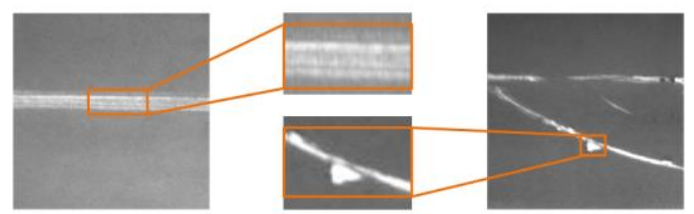

(b)

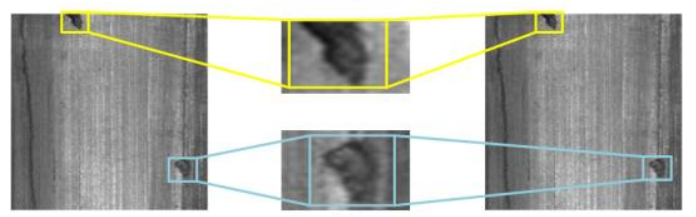

(c)

Fig. 1. Challenges of defect inspection from industry. (a) Defects with lowcontrast. (b) Defects with great difference between intra-class. (c) Defects with similarity between inter-class.

Because of the complexity of defects, there are three main challenges in the automatic defect detection task: 1) lowcontrast: in the industrial production, the existence of dust and the change of light intensity result in the low-contrast between defects and background in the image. Fig. 1 (a) shows that the defects in red box are hardly visible; 2) intra-class difference: unlike other applications, in industrial production, the shape of the defect is irregular. As shown in Fig. 1(b), the multiple scales of defects in the same kind are great different; 3) inter-class similarity: due to the uncertainty of the production process, some different kinds of defects have little difference. Fig. 1(c) presents the different types of defects (in yellow and blue boxes), which are very similar in texture and grayscale information;

Benefiting from the rapid development of computer vision, the above challenges are gradually being addressed in the industrial production. Zhang et al. [1] used curvature filter and gaussian mixture model to the rail surface defect detection. Wang et al. [2] applied template-based methods to the strip surface defect detection. Other approaches based on hand crafted feature are used for defect detection in industrial applications (such as solar modules [3], metal [4], and steel [5]) and have achieved good result in recent years. However, these methods are artificially design a set of features for a specific defect, which isn't universal. 


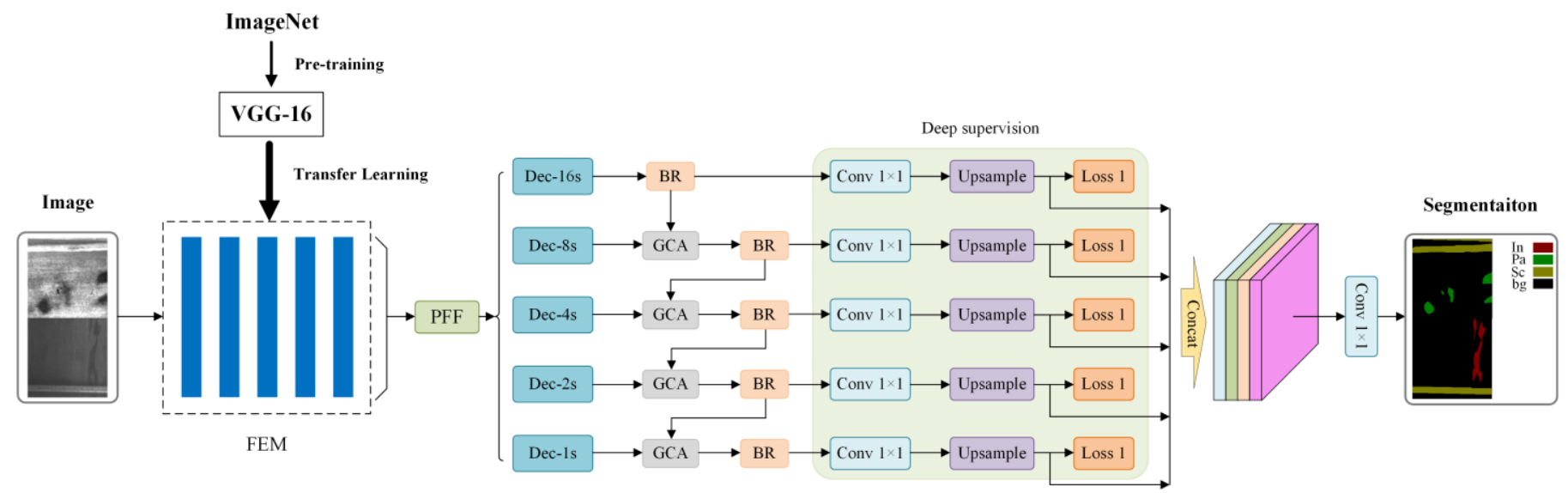

Fig. 2. Architecture of the proposed PGA-Net. Given an input image, we first use the pre-trained CNN to get the feature maps from the last convolutional layer of each stage, then a pyramid feature fusion (PFF) module is applied to fuse these feature maps into five different resolutions, followed by global context attention (GCA) module and boundary refinement (BR) block to combine the adjacent resolutions and refine the predicted maps. Finally, the multiple outputs from boundary refinement are to carry out deep supervised learning. The final prediction is the fused of these multiple outputs.

Recently, deep learning [6] based methods have proven to be effective in many vision tasks. Deep learning that using nonlinear combination, and building convolutional neural network (CNN) architecture [7]-[9] which capacity is controlled by varying the breadth and depth to make strong and substantially correct assumptions about the nature of the image (i.e., the locality of the statistical stationarity and the pixel dependency) has been proved better than artificially design features. Currently, the detection methods based on CNN have been widely used in industrial defect detection, which complete defect detection by designing different network models. For low-contrast, model needs to make effective use of features of object to distinguish the difference between object and background. As [10] indicates, features at different levels of CNN have different sensitivity to the objects. The low-level features have a higher resolution which can generate sharp and detailed boundaries but less context information, while the high-level features have more abstract semantic information which are skilled in making category classification but weak in shape and location. Most methods [11]-[13] mainly focus on the high-level features extracted from deep layers of network. Since the lack of low-level features extracted from shallow layers (e.g., boundary, texture and grayscale information), which lead to poor prediction. Inspired by [14]-[17], this paper utilizes the features extracted from last convolution layer of each stage of backbone network, which include low-level coarse features and high-level semantic features. For intra-class difference, model needs to be undeformed for various changes (such as shape, scale and texture). Most methods based fully convolutional network expand the receptive field to cover the whole defect to achieve the perception of the object change. In [18], dilated convolution (with different dilate rates) is used to expand the receptive field at the last convolution layer of the backbone network to enhance the cognition of the feature change. However, this will cause grid artifacts [19][20]. Zhao et al. [21] fuses features extracted from backbone network under different scales by pyramid pooling module with different pooling kernels. However, pooling will cause the loss of information [22]. To solve these issues» this paper proposes a pyramid feature fusion module, which uses multi-scale convolution (with different size kernels) to weight the feature maps from last convolution layer of each stage of backbone network, so as to obtain the context information of different stages, and then these extracted features of the same resolution are fused at each stage. This not only avoids the gridding artifacts and lack of information, but also the context information is fully extracted. Meanwhile, using the same size strip as the convolution kernel width will not bring large computation. For inter-class similarity, model also be sensitive to the overall transformation, as each pixel needs to be classified in the correct location. In [23], the high-level features are upsampled directly and then fused with low-level features, which is inefficient [24]. Lin et al. [25] obtains the multiple context information and aggregates features from high-level to low-level to refine the features detail, but it will result in a large number of parameters. To address this problem, we add global context attention module to adjacent resolution fusion maps, which extract global context information from low-resolution fusion map then weight highresolution ones to refine the spatial location of category pixel. This not only ensures the effective dissemination of information, but also does not increase the amount of computation.

Based on the improvement of the above theories, this paper carried out extensive experiments on four different defect datasets to show effectiveness and generalization of our approach.

In summary, five major contributions are as follows:

1) A surface defect detection method based on deep learning is introduced, which has achieved state-of-the-art performance on four different surface defect datasets. This proves that the method has certain generality and theoretical value.

2) A method was proposed for surface defect detection at pixel-wise rather than image-level or region-level. Meanwhile, the method aims to detect and distinguish different kinds of defects, not just highline the conspicuous regions in an image.

3) A pyramid feature fusion module was provided, which fuses multi-level features from all stages of backbone CNN into multi-scale resolutions, and learns these resolutions respectively. 
4) A global context attention module was designed, which embedded in these resolutions to ensure efficient information transfer from low-resolution to highresolution.

5) The deep supervision and boundary refinement are added to the proposed method to optimize the network for multibranches, and accelerating convergence during the training process. The final framework achieves outperformance on four defect datasets.

The residue of the paper is organized as follows: Related works about surface defect detection are given in Section II. Next, the proposed PGA-Net is narrated thoroughly in Section III. Afterwards, Section IV describes the evaluation on four defect datasets and corresponding discussions. Finally, the conclusion and suggestions to further improvement are shown in Section V.

\section{RELATED WORKS}

In recent years, the methods based on computer vision for surface defect detection can be categorized into traditional detection approaches and deep-learning-based detection approaches.

\section{A. Traditional detection approaches}

This section refers traditional detection approaches as nodeep learning-based approaches. In the past decade, the traditional approaches can be categorized into statistical-based approaches, filter-based approaches and model-based approaches.

1) Statistical-based approaches: The statistical-based approaches were applied to measure the distribution of pixel values. Popular statistical-based methods usually adopt histogram-of-oriented-gradient, co-occurrence matrix [26], and local-binary-pattern [27] for surface defects detection.

2) Filter-based approaches: Filter-based approaches adopt a bank of filters to describe texture on images in a transformed domain, which are widely used for texture analysis. The filterbased approaches can be categorized into three domains of spatial, frequency [28], and spatial-frequency.

3) Model-based approaches: Model-based approaches obtain certain models with special distributions or other attributes using certain models, which require a high computational complexity [29].

Despite these techniques have achieved good performance on the description of texture features and the detection of texture defects, most of them are applied for homogeneous textures and heavily dependent on expertise.

\section{B. Deep-learning-based detection approaches}

According to different surface defect detection tasks, deep learning-based approaches can be categorized into image-level defect classification, region-level defect inspection, and pixellevel defect segmentation.

Image-level defect classification: Masci et al. [30] proposed a multi-scale pyramidal pooling network for classification of steel defect, which didn't require the size of all images to be equal. Natarajan et al. [31] proposed a flexible multi-layered
TABLE I

DETAILS OF FEATURES EXTRACTION MODULE

\begin{tabular}{|c|c|}
\hline \multirow{3}{*}{ Stage } & Type \\
\hline & 33 conv, stride $=1$ \\
\hline & 22 max pool, stride $=2$ \\
\hline Block1 & $\begin{array}{c}{[\operatorname{conv} 33+\mathrm{BN}+\operatorname{ReLU}, C=64] 2} \\
\max \text { pool } 2 \times 2\end{array}$ \\
\hline Block2 & $\begin{array}{c}{[\operatorname{conv} 33+\mathrm{BN}+\operatorname{ReLU}, C=128]} \\
\max \text { pool } 2 \times 2\end{array}$ \\
\hline Block3 & $\begin{array}{c}{[\operatorname{conv} 33+\mathrm{BN}+\operatorname{ReLU}, C=256]} \\
\max \text { pool } 2 \times 2\end{array}$ \\
\hline Block4 & $\begin{array}{c}{[\operatorname{conv} 33+\mathrm{BN}+\operatorname{ReLU}, C=512]} \\
\max \text { pool } 2 \times 2\end{array}$ \\
\hline Block5 & {$[\operatorname{conv} 33+\mathrm{BN}+\operatorname{ReLU}, C=512]$} \\
\hline
\end{tabular}

deep feature extraction through transfer learning and SVM classifiers, which overcome the problem of over-fitting caused by small datasets. He et al. [32] proposed a semi-supervised model of CNN for feature extraction and fed the representation features into a classifier for classification of steel surface defect. However, these methods can't give the exact location of defects. Meanwhile, when there are many kinds of defects in the image, the accuracy of these methods will also be reduced.

Region-level defect inspection: He et al. [33] proposed a multilevel-feature fusion network, which combined multi-level hierarchical features extracted from a backbone CNN into one resolution for steel plate defect inspection. Chen et al. [34] proposed an approach based on $\mathrm{CNN}$, which analyzed individual video frames for crack detection through CNN and Naïve Bayes data fusion scheme. Zhou et al. [35] improved a deep convolution neural network, which applied a new anchor mechanism to generate suitable candidate boxes for objects, and combines multi-level features to construct discriminative hyper features for split pins defect inspection. The shortcomings of these methods are that they can only provide a coarse region of defects through one or more tight-fitting bounding boxes, but can't describe the defect boundary precisely.

Pixel-level defect segmentation: Currently, the most effective surface defect detection methods are based on the fully convolutional network [11]. A novel CNN was proposed in [36], which integrated context information from top-to-down in a feature pyramid way for pavement crack detection. Ren et al. [37] proposed a deep-learning-based framework for defect classification, then obtain the pixel-wise prediction through the trained classifier convoluted with raw image. Yang et al. [38] proposed a multiscale feature-clustering-based fully convolutional for texture surface defect inspection. Compared with image-level and region-level based methods, the methods based on pixel-level can locate the defect and describe the defect boundary more accurately. However, the results of these methods also need to be improved: i) Most of these methods focus on the high-level features, ignoring the importance of low-level features information. Meanwhile, the output is only one-side prediction, the detect results is poor. ii) Part of these methods adopt more-side prediction, and then fuse these predictions directly to output the final prediction, which lack of the intrinsic relationship of different resolutions feature maps. In contrary, we propose a pyramid feature fusion module to 


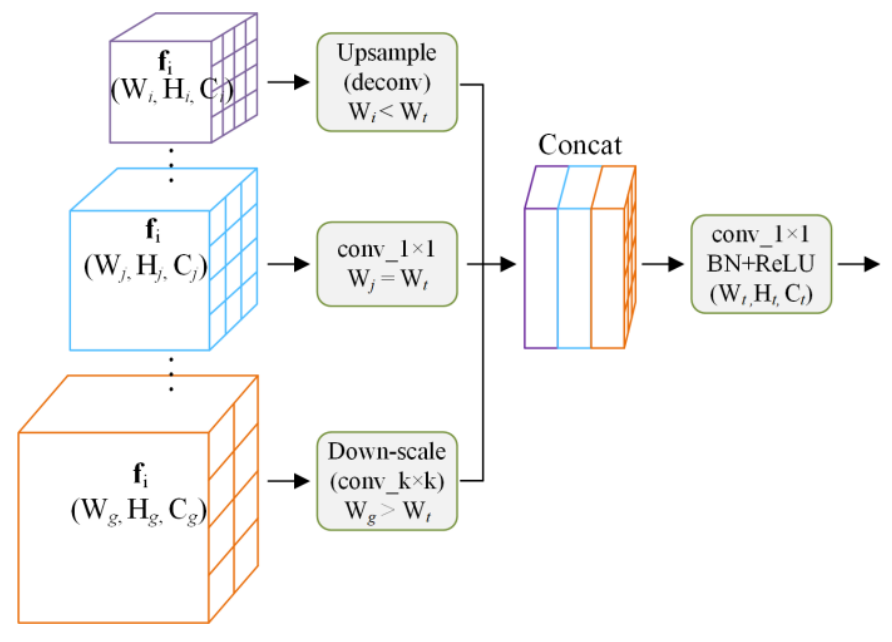

Fig. 3. Details of the PFF module. The PFF module first takes multi-scale features from FEM. Then through the up-scale, down-scale and conv operation to resize the features to the same dimension as the target fusion resolution. Finally, the concatenation and 11 convolution are used to output the final fusion feature maps.

TABLE II

DetaIls of Pyramid FEATURe Fusion Module

\begin{tabular}{|c|c|c|c|c|c|}
\hline Stage & Dec-1s & Dec-2s & Dec-4s & Dec-8s & Dec-16s \\
\hline Conv1_2 & $11, s=1$ & $22, s=2$ & $4 \quad 4, s=4$ & $8 \quad 8, s=8$ & $1616, s=16$ \\
\hline Conv2_2 & deconv & $11, s=1$ & $22, s=2$ & $4 \quad 4, s=4$ & $88, s=8$ \\
\hline Conv3_3 & deconv & deconv & $11, \mathrm{~s}=1$ & $22, s=2$ & $44, s=4$ \\
\hline Conv4_3 & deconv & deconv & deconv & $11, s=1$ & $22, s=2$ \\
\hline Conv5_3 & deconv & deconv & deconv & deconv & $11, s=1$ \\
\hline
\end{tabular}

utilize the feature information of different layers fully. We fuse these features into different resolutions, and adopt global context attention module to fuse them step by step.

\section{Methodology AND DESIGN}

\section{A. System Overview}

In this work, surface defect detection is regard as a pixel-wise task. The architecture of the proposed approach includes five major components: i) feature extract network for multi-level features extraction; ii) pyramid feature fusion module; iii) global context attention module; iv) boundary refinement block; v) deep supervision, as shown in Fig. 2.

1) First, input a batch size of raw images and corresponding ground truth to network, and extract the multi-level features by feature extraction network with convolution and pooling operation. The model learns the effective features in each image of the training samples through forward propagation, and these features correspond to the ground truth one by one to inform the attributes of these features. At the forward propagation, the output feature maps and ground truth are used to calculate the loss. Then back propagation algorithm minimizes the loss and achieves the goal of optimizing the network.

2) Next, feeding these features into pyramid feature fusion module. Adjusting the dimension by convolution and deconvolution (with different kernels and strides) operations to make the fused feature maps have the same dimension. Through some dense skip connects and fuse these features into five resolutions at once.

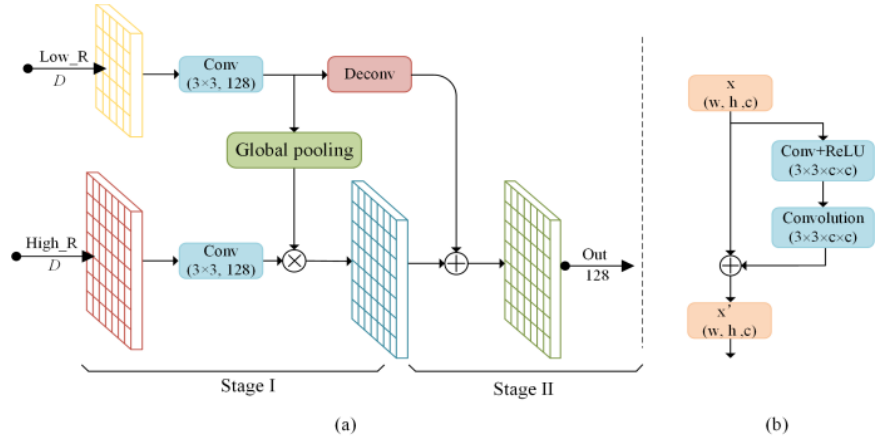

Fig. 4. The details of global context attention module and boundary refinement block are illustrated in (a) and (b), respectively.

3) Then, global context attention embedded in these resolutions to allow for effective information propagate from low-resolution to high-resolution. The output of each global context attention is followed by boundary refinement. Resize the dimension of each resolution to make it same as the raw image to yield prediction maps.

4) Finally, fuse these prediction maps and produces the final prediction.

\section{B. Multi-level Features Extraction Module}

CNNs are widely used to extract features from objects follow their characteristics, and these features can be learned by stacking multiple convolution and pooling layers.

In this paper, the deep feature extraction module (FEM) was built on the VGG-16 [39] model pretrained with ImageNet [40] dataset to extract multi-level features for surface defect detection. The FEM includes five blocks, and these blocks extract appearance information on various, from shallow, fine layers (block_1 and block_2) to deep, coarse layers (block_4 and block_5). Each block consists convolution layers, rectified linear unit activation function (ReLU), batch normalization, and max-pooling layer except the last block. The details of FEM can be referred to Table I, all these layers are optimized by stochastic gradient descent in the process of back propagation to minimize the difference between prediction and ground truth.

\section{Pyramid Feature Fusion Module}

In deep $\mathrm{CNN}$, the extent to how much context information is used roughly depends on the size of receptive field. For defect detection, some defects are intra-class difference and through the whole image (as shown in Fig. 1(b)), which need large receptive field to realize the overall perception of the defect in image. However, the size of actual receptive fields in the CNN are smaller than the theoretical ones [41]. Inspired by [42][43], the pyramid feature fusion (PFF) module was proposed in this paper as shown in Fig. 3, which can be divided into three steps. First, give an input image I with size $W \times H$, and through FEM module generates multi-level features at different stages. The PFF module obtain last layer feature of each stage: conv1_2, conv2_2, conv3_3, conv4_3, conv5_3. For simplicity, these five features could be denoted by a feature set $\boldsymbol{F}$ : $\boldsymbol{F}=\left(\mathbf{f}_{1}, \mathbf{f}_{2}, \mathbf{f}_{3}, \mathbf{f}_{4}, \mathbf{f}_{5}\right)$, where $\mathbf{f}_{1}$ denotes the conv1_2 features and 
so on. Second, multi context information is generated by multiscale receptive fields weighted $\boldsymbol{F}$, and this information is mapped to five different resolution feature maps at the same time: $T_{n}=\left(W / 2^{n}, H / 2^{n}\right)$, where $n=(0,1,2,3,4), W$ and $H$ represent the width and height of the input image, respectively. For $\mathbf{f}_{1}$ (resolution $R_{I}=T_{0}$ ), the module down-scales it to five resolution with a stack of convolution layers, and the output feature maps $\boldsymbol{Y}_{1}^{i}$ as follows:

$\boldsymbol{Y}_{1}^{i}=\mathrm{F}\left(\mathbf{f}_{1} \mid \boldsymbol{W}, \boldsymbol{b}\right)=\boldsymbol{s}\left(\right.$ down-scale $\left.\left(\boldsymbol{W}_{k \text { ks }} \quad \mathbf{f}_{1} \quad \boldsymbol{b}\right)\right)\left(\begin{array}{lll}i & 7, & , 5\end{array}\right)$

where $\sigma$ refers to the ReLU activation, down-scale $(\cdot)$ signifies through $\boldsymbol{W}_{k k}$ (kernel size is $k \times k$, stride $s=k$ ) to downscale the feature map $\mathbf{f}_{1}, \boldsymbol{b}$ denote bias, denotes convolution. For $\mathbf{f}_{5}$ (resolution $R_{5}=T_{4}$ ), the module upsamples it into five resolution, the output feature maps $\boldsymbol{Y}_{5}^{i}$ as follows:

$$
\boldsymbol{Y}_{5}^{i}=\mathrm{Y}\left(\mathbf{f}_{5}\right)=\boldsymbol{s}\left(\text { upsample }\left(\mathbf{f}_{5} ; y\right)\right)(i=1, \quad, 5)
$$

where $\sigma$ refers to the ReLU activation, upsample $(\cdot ; \psi)$ refers the deconvolution with parameters $\psi$ which are learned during the training. For $\mathbf{f}_{2}, \mathbf{f}_{3}$ and $\mathbf{f}_{4}$, which resolution between $T_{0}$ and $T_{4}$, the model uses the combination of down-scale and upsample to resize them into five resolution, and the output feature maps $\boldsymbol{Y}_{l}^{i}$ as follows:

$$
\boldsymbol{Y}_{l}^{i}\left(\mathbf{f}_{l}\right)=\boldsymbol{s}\left(\mathrm{F}\left(\mathbf{f}_{l}\right) \& \mathrm{Y}\left(\mathbf{f}_{l}\right)\right)(i=1, \quad, 5 ; l \quad 2,3 ; 4)
$$

where $\sigma$ refers to the ReLU activation, $\Phi(\cdot)$ and $\Psi(\cdot)$ denote Equa. (1) and Equa. (2), respectively. The channel dimension of these resized feature maps $\left(\boldsymbol{Y}_{1}^{i}, \quad, \boldsymbol{Y}_{5}^{i}\right)$ is 128 . Finally, the features with same dimension in these output ones are fused to generate the final five fused feature maps. To be convenient, the five fused feature maps are respectively named Dec-1s $(\mathrm{n}=0)$, Dec-2s ( $(n=1)$, Dec-4s $(n=2)$, Dec-8s ( $n=3)$, Dec-16s $(n=4)$. The five fused features could be defined as:

$$
\operatorname{Dec}^{-i_{s}}=\boldsymbol{s}\left(\begin{array}{lllll}
\boldsymbol{W}_{11} & \boldsymbol{C A T}\left(\boldsymbol{Y}_{1}^{i},\right. & \boldsymbol{Y}_{3}^{i}, & \left.\boldsymbol{Y}_{5}^{i}\right) & \boldsymbol{b}
\end{array}\right)
$$

Where $\sigma$ refers to the ReLU activation, CAT denotes the element-wise concatenated. The channel of each fused features map Dec is $640 . \boldsymbol{W}_{11}$ denotes a convolution with $1 \times 1$ kernel size to change the channel dimension of concatenated features (640 to 128), $b$ refers bias. All convolution layers defined in PFF are followed by ReLU activation and Batch Normalization and these parameters are trainable, as shown in Table II. Through this way, the model effectively obtains the multi-scale context information from different stages of $\mathrm{CNN}$, and realizes the overall perception of the object.

\section{Global Context Attention Module}

The final fusion feature maps with different resolution generated from PFF contains various visual context information, and each of them can be used to yield the result prediction. One method using bilinear upsample to up-scale these fused features into the same dimension with the raw image, then change their channel through a convolutional layer to the number of classes to predict the segmentation result. However, the shortcomings of these approaches are: i) they lack the inner relation information between different resolution predictions, ii) use bilinear upsample with a big kernel directly may lead to the missing of some detail information and the parameters are not trainable. Other U-shape models [23][44] combine the adjacent feature maps from low-resolution to high-resolution step-bystep in the decoding process. However, there are also two shortcomings in these methods: i) The type of this combination between adjacent features maps in the decoding process is too single and lack diverse representation, ii) lack the global context information from low-resolution (high-level), which can enhance high-resolution (low-level) feature map in decoding process.

To address above issues, a global context attention module (shown in Fig. 4(a)) was proposed, which consists of two stages:

Stage one: A $3 \times 3$ convolution was applied to adjust the channels dimension of high-resolution and low-resolution fusion feature maps, then through global pooling to the lowresolution to obtain global context, following multiplied with the high-resolution feature map. The output $f_{\mathrm{s} 1}$ as follows:

$$
\left.f_{s 1}=s\left(\begin{array}{lll}
\boldsymbol{W}_{33} & f^{h} & b
\end{array}\right) \quad s\left(G\left(\begin{array}{lll}
\boldsymbol{W}_{33} & f^{l} & \boldsymbol{b}
\end{array}\right)\right)\right)
$$

where and $*$ denote element-wise multiplication and convolution, respectively, $G()$ denotes global pooling operation, $\sigma$ refers to ReLU activation, $f^{h}$ and $f^{l}$ represent highresolution and low-resolution fusion feature maps, $\boldsymbol{W}_{3}$ indicates trainable parameters, $b$ refers bias.

Stage two: The low-resolution fusion feature map is upsamped to the same dimension with the high-resolution, and then added with the $f_{\mathrm{s} 1}$. The output of stage two $f_{\mathrm{s} 2}$ as follows:

$$
f_{s 2}=s\left(\text { upsample }\left(f^{l} y\right) f_{s 1}\right)
$$

Where upsample $(\cdot ; \psi)$ refers the deconvolution with parameters $\psi$ which are learned during the training, refers to element-wise addition.

In short, compared with simply adding the upsampled coarser-resolution feature maps to the finer-resolution ones, the proposed GCA module can utilize different resolution fusion feature maps to improve the efficiency of context obtain and corresponding pixel-wise localization.

\section{E. Boundary Refinement Block}

In this paper, we add boundary refinement [45] block to further improve the detection accuracy, shown in Fig. 4(b). The boundary refinement was seen as a residual structure, the output refined score map $\tilde{s}$ as follows:

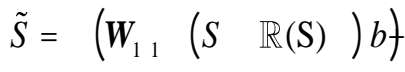




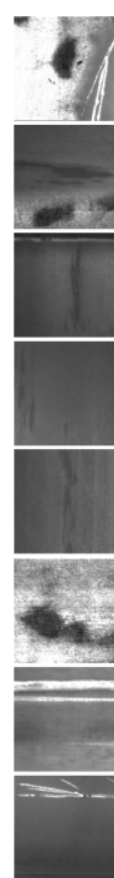

(a)

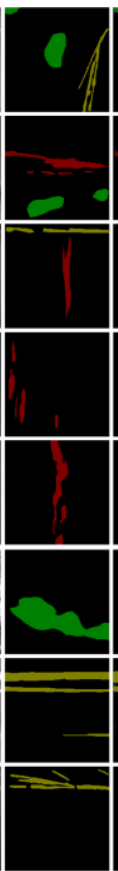

(b)

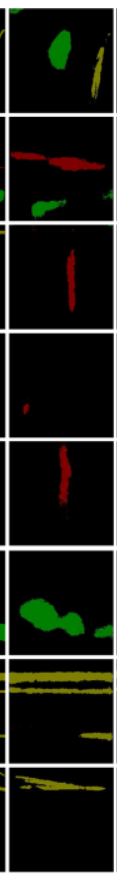

(c)

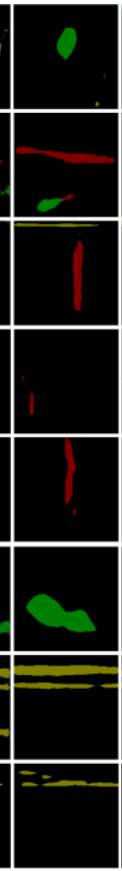

(d)

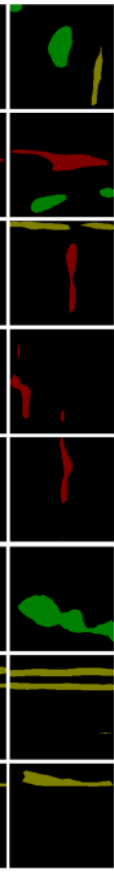

(e)

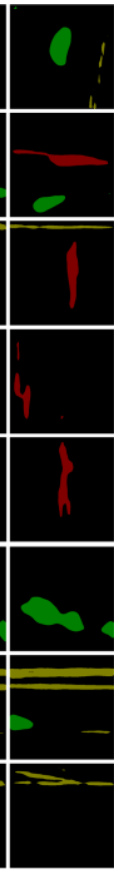

(f)

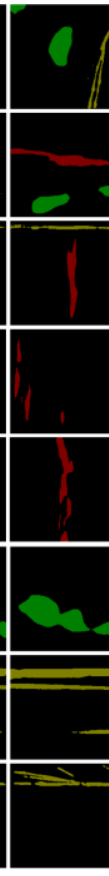

(g)

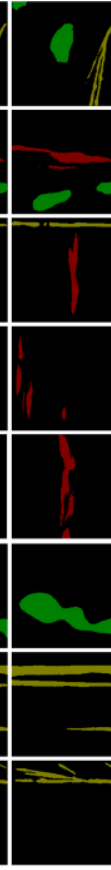

(h)
Fig. 5. Comparison of detection results on NEU-Seg dataset. Red, green and yellow represent inclusion (In), patches $(\mathrm{Pa})$ and scratches $(\mathrm{Sc})$ defect, respectively. (a) Original image. (b) Ground truth. (c) SegNet. (d) PSPNet. (e) DeepLab. (f) RefineNet. (g) FCN. (h) PGANet

TABLE III

QUANTITATIVE COMPARISONS OF DIFFERENT DETECTION METHODS

\begin{tabular}{c|lcc}
\hline Dataset & Method & mIoU (\%) & Time (s) \\
\hline \multirow{5}{*}{ NEU-Seg Dataset } & SegNet [23] & 56.57 & 0.0528 \\
& PSPNet [21] & 72.25 & 0.0375 \\
& DeepLab [18] & 74.01 & 0.0104 \\
& RefineNet [25] & 75.37 & 0.0315 \\
& FCN [11] & 81.79 & 0.0665 \\
& PGA-Net & 82.15 & 0.0206 \\
\hline
\end{tabular}

where $\mathrm{S}$ and $\mathbb{R}(\cdot)$ signify the coarse score map and residual branch, respectively, $*$ represents convolution, $\sigma$ refers to the ReLU activation, is the cross-channel concatenation, $b$ refers bias. The details were shown in Fig. 4(b).

\section{F. Deep supervision}

Although multi-level features are fully utilized, the mount of parameters is also increased obviously, which may introduce additional optimization difficulty. To address the issue, we add deep supervision into our model, which aims to ease the process of training and accelerate the optimization of network model.

The fused feature maps generated from PFF module at each resolution can performs crack prediction individually. We add a per-pixel loss (cross-entropy) to each of the above five resolution fused maps. The loss function is described as:

$$
\boldsymbol{L}(T, P)=-\frac{1}{N}{ }_{i=1}^{N} T_{i} \log P_{i} \quad\left(1-T_{i}\right) \log \left(\begin{array}{ll}
1 & -P_{i}
\end{array}\right)
$$

where $T_{i}$ and $P_{i}$ represent ground truth and predicted probabilities of $i^{\text {th }}$ image, respectively, $N$ refers batch size. In the test phase, the predictions generated from the five branches are fused to output the result of detection, as shown in Fig. 2.

\section{EXPERIMENTS AND RESULTS}

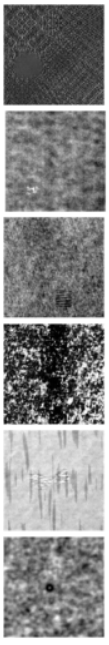

(a)

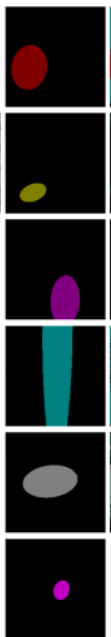

(b)

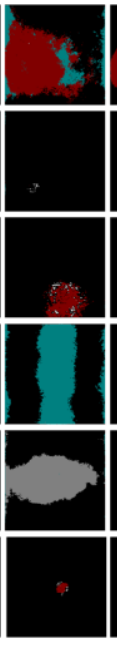

(c)

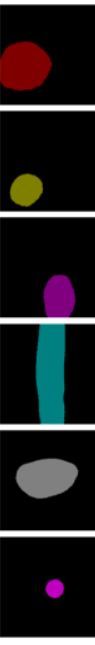

(d)

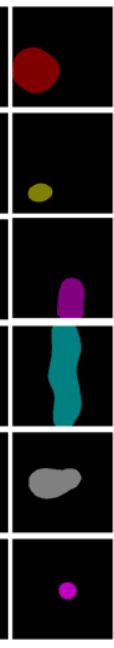

(e)

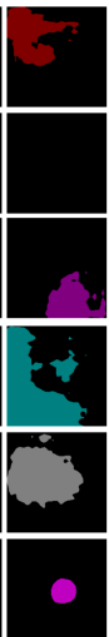

(f)
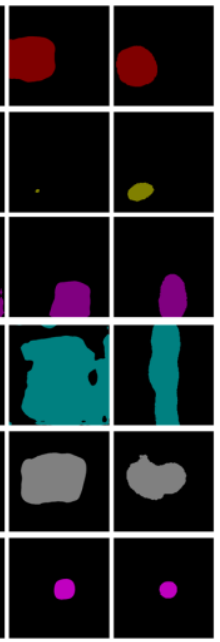

(g)

(h)

Fig. 6. Comparison of detection results on DAGM 2007 dataset. (a) Original image. (b) Ground truth. (c) SegNet. (d) FCN. (e) DeepLab. (f) PSPNet. (g) RefineNet. (h) PGA-Net

TABLE IV

QUANTITATIVE COMPARISONS OF DIFFERENT DETECTION METHODS

\begin{tabular}{c|lcc}
\hline Dataset & Method & mIoU (\%) & Time (s) \\
\hline & SegNet [23] & 21.95 & 0.0558 \\
& RefineNet [25] & 32.90 & 0.0322 \\
DAGM 2007 & PSPNet [21] & 41.21 & 0.0369 \\
Dataset & FCN [11] & 73.86 & 0.1041 \\
& DeepLab [18] & 74.61 & 0.0108 \\
& PGA- Net & 74.78 & 0.0229 \\
\hline
\end{tabular}

TABLE V

\begin{tabular}{l|cc}
\multicolumn{3}{c}{ THE NUMBER OF FOUR DATASETS } \\
\hline Dataset & Train & Test \\
\hline NEU-Seg & 3630 & 840 \\
DAGM 2007 & 3550 & 400 \\
MT Defect & 2840 & 300 \\
Road Defect & 6000 & 400 \\
\hline
\end{tabular}

\section{A. Implementation Details}

1) Parameters Setting: The initialization parameters of FEM are transformed from the pre-trained VGG-16 network which for image classification task on the ImageNet dataset. Furthermore, the weights realize initialization of other convolutional layers through "Xavier" scheme. As for up-scale features, we use transposed convolution with learnable weights. For fine-tuning, we set the base learning rate is $10^{-5}$ with a decay of 0.005 , the max-inter is $100 \mathrm{k}$ with mini-batch size 5 . The model is saved every 5,000 iterations.

2) Computation Platform: We implement our method on the PyCharm with the open source toolbox TensorFlow [46]. We run our method in a NVIDIA GTX TITAN GPU (with 12G memory) on Ubuntu 16.04 Linux.

\section{B. Datasets}

1) Datasets Description: In this work, four surface defect datasets are selected to prove and evaluate the applicability and generality of the proposed method, including NEU-DET defect dataset, DAGM 2007 defect dataset, MT defect dataset and Road defect dataset.

NEU-Seg Dataset: NEU-Seg defect dataset is a standardized high-quality database, which collected by [51] to solve the problem of automatic recognition for hot-rolled steel strip. This 


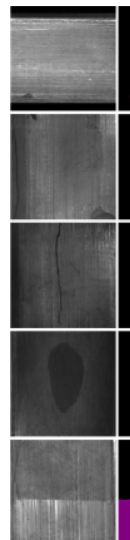

(a)

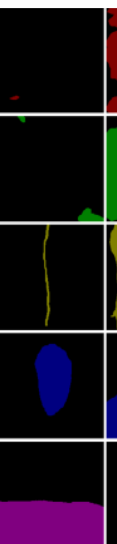

(b)

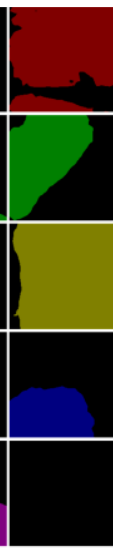

(c)

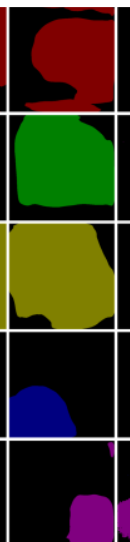

(d)

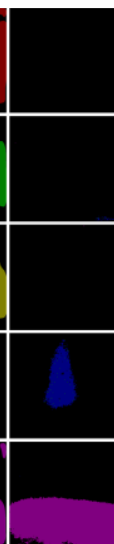

(e)

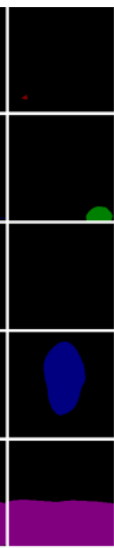

(f)

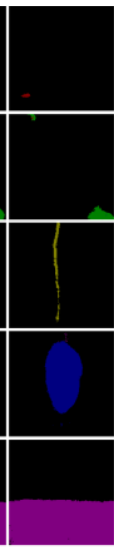

(g)

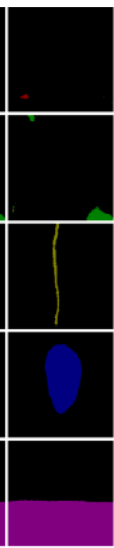

(h)
Fig. 7. Comparison of detection results on MT defect dataset. Different color represent different kinds of defects, respectively. (a) Original image. (b) Ground truth. (c) PSPNet. (d) RefineNet. (e) SegNet. (f) DeepLab. (g) FCN. (h) PGA-Net

TABLE VI

QUANTITATIVE COMPARISONS OF DIFFERENT DETECTION METHODS

\begin{tabular}{c|lcc}
\multicolumn{4}{c}{ QUANTITATIVE COMPARISONS OF DIFFERENT DETECTION METHODS } \\
\hline Dataset & Method & mIoU (\%) & Time (s) \\
\hline \multirow{4}{*}{ MT Defect } & PSPNet [21] & 12.84 & 0.0401 \\
Dataset & RefineNet [25] & 13.52 & 0.0335 \\
& SegNet [23] & 33.32 & 0.0550 \\
& DeepLab [18] & 49.21 & 0.0124 \\
& FCN [11] & 67.83 & 0.2735 \\
& PGA- Net & 71.31 & 0.0246 \\
\hline
\end{tabular}

dataset includes six categories surface defects from strip steel plates, including patch, crazing, pitted-surface, inclusion, scratches, and rolled-in scale. The resolution of each raw image is $200 \times 200$ and each class include 300 images with tightfitting bounding box annotations. However, in order to achieve the pixel-wise surface defect detection task, this form of annotation does not satisfy the training of CNN model. In this work, three typical defects (inclusion, patch and scratches) are selected, and conduct pixel-wise annotation by the open annotation tool: LabelMe. This dataset is named as NEU-Seg datasets. Due to the complexity of the situation of hot-rolled plates, there are large differences in appearance of the defects between intraclass, while the defects between inter-class contain similar parts, as well as the low contrast with background. All these factors bring great challenges to the surface defect detection of hotrolled strip steel. Figure 5 shows the visualization of partial NEU-Seg raw images and corresponding ground truth.

DAGM 2007 Dataset: This dataset [47] which produced by artificial represents defects under a textured background is very close to real-world. This dataset includes many categories defects and the resolution of each raw image is $512 \times 512$. In the label images of DAGM 2007, the defect regions are blanket roughly by ellipses. In this experiment, six types of defects are selected and redefine the raw label (we didn't change the size of the raw defect area, just changed the index in the label image), the different indexes in the new label image represent different categories. Figure 6 shows partial defect images and corresponding ground truth of DAGM 2007 datasets.

MT Defect Dataset: The magnetic-tile defect dataset is presented in [48] which contains 1344 defect images, and each raw defect image corresponds to a pixel-level label. MTdefect dataset includes five types of defects: uneven, fray, crack,

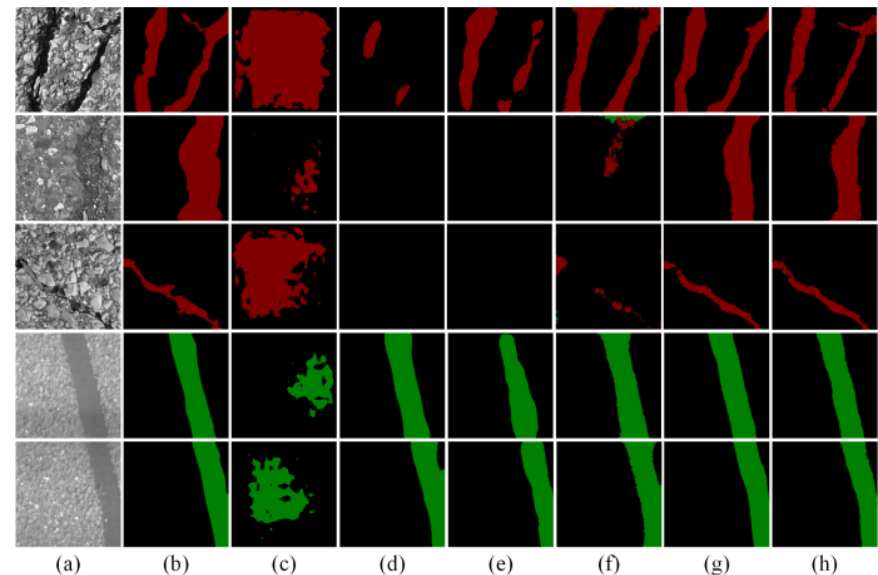

(a)

(b)

(c)

(d)

(e)

(f)

(g)

(h)

Fig. 8. Comparison of detection results on Road defect dataset. Red and green represents crack and inlaid patch, respectively. (a) Original image. (b) Ground truth. (c) PSPNet. (d) DeepLab. (e) RefineNet. (f) SegNet. (g) FCN. (h) PGANet

TABLE VII

QUANTITATIVE COMPARISONS OF DIFFERENT DETECTION METHODS

\begin{tabular}{c|lcc}
\hline Dataset & Method & mIoU (\%) & Time (s) \\
\hline \multirow{5}{*}{ Road Defect } & PSPNet [21] & 27.65 & 0.0374 \\
Dataset & RefineNet [25] & 46.21 & 0.0322 \\
& DeepLab [18] & 47.63 & 0.0109 \\
& SegNet [23] & 65.04 & 0.0568 \\
& FCN [11] & 78.74 & 0.1018 \\
& PGA- Net & 79.54 & 0.0218 \\
\hline
\end{tabular}

blowhole, and break, all these defect images with different resolution. Most of these defect images contain a series of noise, e.g., the diversity of defect shape, complexity of texture, and the change of illumination intensity, all these factors bring a big challenge of detection. In this experiment, we detect five types defects (blowhole, crack, fray, break and uneven) of magnetictile defect dataset. Figure 7 shows the partial raw defect images and corresponding ground truth.

Road Defect Dataset: This dataset contains two classes (crack, inlaid patch). The number of crack images are 500 with size around 2,000 1,500 pixels, which collected by [36]. The inlaid patch images that we collect by CCD contains 800 images of size around 3,000 2,000. Each defect image corresponds to a pixel-level label with different indexes. In this experiment, these raw images are randomly cut to 256256 to improve diversity of the dataset, the effective area of the defect in each crop-image is kept above twenty percent. Figure 8 shows example raw defect images and corresponding ground truth.

2) Data Augmentation: Deep learning-based detection methods usually require a great many datasets to overcome over-fitting during training. However, in the actual production process, it is difficult to collect a large of high-quality defect image due to factors such as environment and equipment etc. The basic method to solve the over-fitting issue caused by the lack of dataset during training the model is data augmentation. In the early stage of the experiment, we cut all the images of four datasets with $200 \times 200$ resolution. Then we rotate the cropped images $\left(90^{\circ}, 180^{\circ}, 270^{\circ}\right)$ to increase the training samples, and the corresponding ground truth are also processed in the same way. To ensure the validity of the samples, samples with defect area less than $10 \%$ of the whole image are deleted. 


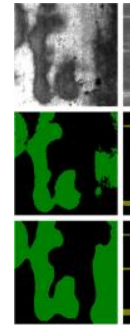

(a)

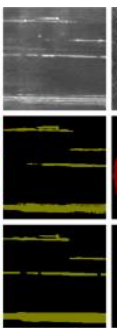

(b)

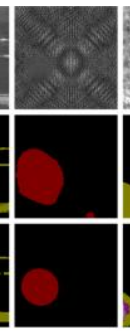

(c)

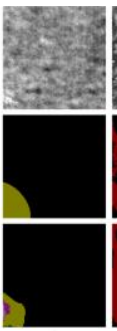

(d)

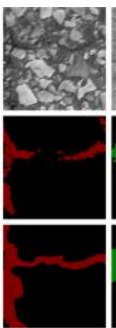

(e) (f)

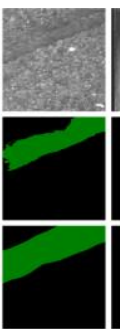

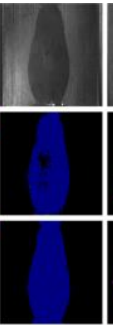

(g)

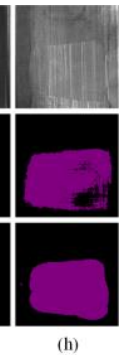

(h)
Fig. 9. The failure of proposed method PGA-Net on four datasets. (a) and (b) are failure detect of NEU-Seg dataset, (c) and (d) are failure detect of DAGM 2007, (e) and (f) are failure detect of Road Defect dataset, (g) and (h) are failure detect of MT Defect dataset.

The details are shown in Table V. Inspired by [40][44], in the process of training, the network randomly extracts $192 \times 192$ size areas (and their horizontal reflections) from input images, then train the framework on these extracted areas, which the training samples are increased 128 times. Although the random extract operation will not change the structure of the object in the image too much, so that each extracted region has high similarity in the object itself, but the operation can change the spatial position information and the semantic information of the target, so as to improve the number and diversity of dataset and avoid over-fitting.

\section{Evaluation Metrics}

Compared with other segmentation methods, mean intersection-over-union (mIoU) is used to performance the evaluation of the prediction result. The mathematical definitions are shown in [11]. We also use the average running time of process each image in this experiment to show the realtime performance of the proposed approach.

\section{Experiment Results and Analysis}

1) Detection Results on NEU-Seg Defect: The visual comparison of our approach and other methods for strip steel surface defect images are shown in Fig. 5. It can be observed that the proposed PGA-Net outstanding the performance than other methods in the challenging cases of defect detection, e.g. low-contrast (the 4-5 rows) and intra-class difference (the 7-8 rows), and the results of prediction are very similar to the ground truth. As the quantitative comparisons shown in Table III, the proposed approach is superior to other compared counterparts in term of the evaluation metrics: the value of mIoU is improved to $82.15 \%$.

2) Results of DAGM 2007 Dataset: The comparison of visual results of partial DAGM 2007 defect images detection shown in Fig. 6. The main detection challenging for this dataset is lowcontrast between the backgrounds and defects (the 1-3 rows). It can be found that [23] miss or erroneously detects some defects. For some large area defects, [21] and [25] can't accurately locate defects. [11] and [18] magnify some low-contrast defect areas. In Contrary, the performance of proposed PGA-Net is closer to the real situation. As listed in Table IV, PGA-Net improves performance to $74.78 \%$.

3) Detection Results on MT Defect: Figure 7 shows some samples of magnetic tile defects and the corresponding visual prediction. The main challenging of this dataset is inter-class similarity (the 1-3 rows). It can be observed from Figure 7 that
TABLE VIII

Detailed PeRformance of OUR METHOD WITH DifFERENT SETTINGS

\begin{tabular}{|c|c|}
\hline Method & $\mathrm{mIoU}(\%)$ \\
\hline Dec-16s & 76.84 \\
\hline Dec-8s & 80.36 \\
\hline Dec-4s & 81.68 \\
\hline Dec-2s & 82.00 \\
\hline Dec-1s & 82.07 \\
\hline $\mathrm{PFF}\left(\operatorname{Conv}_{k \times k}\right)+\mathrm{GCA}$ & 80.46 \\
\hline PFF(Max pooling) + GCA & 79.89 \\
\hline PFF $\left(\operatorname{Conv}_{k \times k}\right)+$ GCA + BR & 82.15 \\
\hline
\end{tabular}

[25] and [21] are failure of predict result. [23] and [18] can locate and detect the defects (the region of defects is large and obvious), but they are easy to miss the defect detection in small area. [11] can effectively defects small defects, but the detected defect area is incomplete. However, the proposed PGA-Net achieves best performance in above aspects. In Table VI, PGANet improves the mIoU to $71.31 \%$.

4) Detection Results on Road Defect Dataset: Figure 8 demonstrates part of the road defect images and the corresponding prediction results. The main challenging cases of this dataset are low-contrast (the 2 row) and intra-class difference (the 1-3 rows). It can be observed from Figure 8 that [21] can detected defects roughly, but failed to locate the region of defect. For crack defects which are low contrast to the background, [25] and [18] failed to detect these defects, [23] and [11] lack of integrity in these defects. As listed in Table VII, the proposed PGA-Net increases the mIoU by $0.8 \%$ compared with the second best.

5) Analysis of Time to Test Each Image: Table III, IV, VI, VII list the average running time to process each image of different methods, and performed on four datasets using a computer introduced in Section IV-A. Compared with other state-of-the-art methods, the time of test each image of our method is not the shortest, but the speed can reach 41-49 fps/s, which is acceptable in the real detection process and does not harm the user experiences. In future research, we will further optimize the code to accelerate the proposed method to meet the needs of real-time and high accuracy.

6) Analysis of Failure cases: The experiment results show that the proposed approach outperforms state-of-the-art detection methods on the four datasets. However, some difficult images still posed challenged to our method as well as those comparative methods. As shown in second line images (a), (b), (e), (g), (h) of Fig. 9, we can see that our method is lack of integrity in detection the defect area of partial defect images. Because of the network over fits the benchmark data, when the difference between the test sample and the training sample is large, it will lead to missed detection. The main reason is the lack of dataset. Meanwhile, the generalization ability of network model needs to be improved. As shown in (c), (d) of the second line of Fig. 9, some defects are detected by mistake. The network model is over sensitive to image changes. When the defect area changes obviously, the network does not regard it as a whole. The main reason for this case is the lack of diversity and number of datasets. We will work on these problems in the future. 


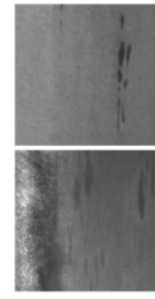

(a)

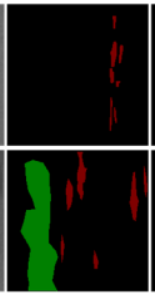

(b)

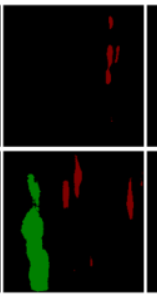

(c)

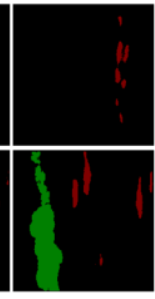

(d)

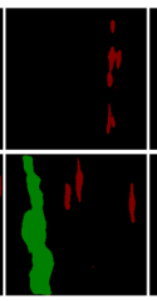

(e)

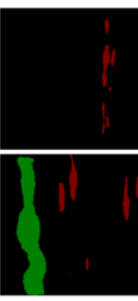

(f)
Fig. 10. Comparison of detection results on NEU-Seg dataset. (a) Original image. (b) Ground truth. (c) UN. (d) DLA. (e) DSS. (f) Ours

TABLE IX

Detailed Performance of DifFerent Multi-SCALE Features Fusion

\begin{tabular}{l|cccc}
\multicolumn{5}{c}{ MoDULES } \\
\hline Method & UN & DLA & DSS & Ours \\
\hline mIoU $(\%)$ & 81.43 & 81.58 & 81.66 & 82.15 \\
\hline
\end{tabular}

\section{E. Ablative Study}

To evaluate the proposed method, this work conducts a rank of ablation experiments, including down-scale type, the effects of fusion feature resolution, and the boundary refinement for detection result. All the evaluate of these ablative experiments based on NEU-Seg dataset.

1) The ablation studies for the down-sacle type: For the down-scale structure in PFF module, this paper uses large kernel convolution ( $\operatorname{conv}_{\mathrm{k}}$ ) to replace max pooling. On the one hand, convolution reduces the dimension of feature and retains feature information, while the max pooling may lead to a large number of feature information loss. Meanwhile, using large kernel size doesn't bring too much computation burden. As shown in Table VIII, the performance is improved from $79.89 \%$ to $80.46 \%$.

2) The effects of fusion feature resolution: Some fused feature structures (spatial and semantics) from FEM are easily destroyed when these features resolution are adjusted by convolution and deconvolution (with big kernel size and stride) which are very different from the resolution of target fusion feature map (Block5_3 $\rightarrow$ Dec-1s / Block1_2 $\rightarrow$ Dec-16s). To verify the effects of fusion feature resolution, this work additionally evaluates the five fusion feature maps (Dec-1s, Dec-2s, Dec-4s, Dec-8s, Dec-16s) come from PFF, and the corresponding performance are shown in Table VIII. As can be seen from table that with the increment of resolutions, the model performance better gradually, which demonstrate the necessary of selection of resolution for feature maps fusion for our method.

3) The ablation of boundary refinements for inspection result: Boundary refinement block is added into the proposed method to improve the performance of result. As shown in Table VIII, with the boundary refinement block yields results $82.15 \%$ in terms of mIoU, which proves the validity of BR for our method.

\section{4) Compared with Other Multi-level Features Fusion} Methods

To verify the advantage of the proposed PFF module, we compared other multi-level features fusion methods, including UN [44], DLA [49], DSS [50]. We replace the PFF module of our proposed method with the multi-level fusion module of these methods. All the modules are based on the same backbone network VGG-16 network. In the experiment, we optimize the parameters of each multi-level features fusion module to achieve the best results. The evaluate results of these compared module on NEU-Seg dataset. The visual comparison of our approach and other methods is shown in Fig. 10. The quantitative evaluation is listed in Table IX. From Fig. 10 and Table IX we can see that compared with multi-level features fusion module, our PFF achieves better performance.

\section{CONCLUSION}

In this work, an automatic defect detection network for surface defect detection is proposed. In the framework, multilevel features from defect images are extracted by feature extraction module. Pyramid feature fusion module is introduced to fuse these multi-level features into different resolutions. Global context attention module makes the effective information propagate from low-resolution fusion feature maps to high-resolution fusion ones. The boundary refinement block is added in the framework to refine the object boundary prediction. Deep supervision is applied in the framework to speed up the process of network optimization. Experiments demonstrate that the proposed approach significantly advances the state-of-the-art approaches on four surface defect datasets detection. However, due to the limitation of the number and diversity of datasets, some defects are missing and wrong detected as shown in Fig.9. Although the speed of detection can reach 41-49 fps/s, which is acceptable in the real detection process and does not harm the user experiences, the detection speed needs to be further improved to meet the needs of realtime and high accuracy. In addition, all the defect samples need to be labeled but consuming time.

In future research, we plan to seek an efficient data augmentation strategy combined with our approach to improve the detection performance, and optimize the framework to accelerate the proposed approach to meet real-time and high accuracy requirement. In addition, semi-supervised mechanism will be adopted in our future work.

\section{REFERENCES}

[1] H. Zhang, X. Jin, Q. M. J. Wu, Y. Wang, Z. He and Y. Yang, "Automatic visual fetection system of railway surface defects with curvature filter and improved gaussian mixture model," in IEEE Trans. on Instrum. and Meas., vol. 67, no. 7, pp. 1593-1608, July 2018.

[2] H. Wang, J. Zhang, Y. Tian, H. Chen, H. Sun and K. Liu, "A Simple Guidance Template-Based Defect Detection Method for Strip Steel Surfaces," in IEEE Trans. on Ind. Informat., vol. 15, no. 5, pp. 27982809, May 2019.

[3] D. Tsai, S. Wu and W. Chiu, "Defect Detection in Solar Modules Using ICA Basis Images," in IEEE Trans. on Ind. Informat., vol. 9, no. 1, pp. 122-131, Feb. 2013.

[4] J. Wang, Q. Li, J. Gan, H. Yu and X. Yang, "Surface Defect Detection via Entity Sparsity Pursuit with Intrinsic Priors," in .2019. DOI: 10.1109/TII.2019.2917522.

[5] Q. Luo, Y. Sun, P. Li, O. Simpson, L. Tian and Y. He, "Generalized Completed Local Binary Patterns for Time-Efficient Steel Surface Defect Classification," in IEEE Trans. on Instrum. and Meas., vol. 68, no. 3, pp. 667-679, March 2019. 
[6] Y. LeCun Y. Bengio G. Hinton "Deep learning” Nature vol. 521 no. 7553 pp. 436-444 May 2015.

[7] K. Jarrett, K. Kavukcuoglu, M. Ranzato and Y. LeCun, "What is the best multi-stage architecture for object recognition?," in , Kyoto, 12th, 2009, pp. 2146-2153.

[8] S.C. Turaga, J.F. Murray, V. Jain, F. Roth, M. Helmstaedter, K. Briggman, W. Denk, and H.S. Seung, "Convolutional Networks Can Learn to Generate Affinity Graphs for Image Segmentation," vol. 22, pp. 511-538, 2010.

[9] Y. Bengio, A. Courville and P. Vincent, "Representation Learning: A Review and New Perspectives," in , vol. 35, no. 8, pp. 1798-1828, Aug. 2013.

[10] A. Mahendran and A. Vedaldi, "Understanding deep image representations by inverting them," 2015 IEEE Conf. on Comput. Vis. and Pattern Recognit. (CVPR), Boston, MA, 2015, pp. 5188-5196.

[11] J. Long, E. Shelhamer and T. Darrell, "Fully convolutional networks for semantic segmentation,"

, Boston, MA, 2015, pp. 3431-3440.

[12] Guanbin Li and Y. Yu, "Visual saliency based on multiscale deep features,"

, Boston, MA, 2015, pp. 5455-5463.

[13] R. Zhao, W. Ouyang, H. Li and X. Wang, "Saliency detection by multicontext deep learning,"

$$
\text { , Boston, MA, 2015, pp. 1265-1274. }
$$

[14] P. Zhang D. Wang H. Lu H. Wang B. Yin "Learning uncertain convolutional features for accurate saliency detection" in Proc. ICCV, Oct. 2017, pp. 212-221.

[15] P. Zhang, D. Wang, H. Lu, H. Wang, and X. Ruan, "Amulet: Aggregating multi-level convolutional features for salient object detection," Proc. ICCV, Oct. 2017, pp. 202-211.

[16] R. Wu, M. Feng, W. Guan, D. Wang, H. Lu, and E. Ding, "A Mutual Learning Method for Salient Object Detection with Intertwined MultiSupervision," 2019 IEEE Conf. on Comput. Vis. and Pattern Recognit. (CVPR), 2019, pp. 8150-8159.

[17] L. Wang, L. Wang, H. Lu, P. Zhang and X. Ruan, "Salient Object Detection with Recurrent Fully Convolutional Networks," in IEEE Trans. on Pattern Anal. and Mach. Intell., vol. 41, no. 7, pp. 1734-1746, 1 July 2019.

[18] L. Chen, G. Papandreou, I. Kokkinos, K. Murphy and A. L. Yuille, "DeepLab: Semantic Image Segmentation with Deep Convolutional Nets, Atrous Convolution, and Fully Connected CRFs," in IEEE Trans. on Pattern Anal. and Mach. Intell., vol. 40, no. 4, pp. 834-848, 1 April 2018.

[19] F. Yu, V. Koltun and T. Funkhouser, "Dilated Residual Networks," 2017 IEEE Conf. on Comput. Vis. and Pattern Recognit. (CVPR), Honolulu, HI, 2017, pp. 636-644.

[20] P. Wang et al., "Understanding Convolution for Semantic Segmentation," 2018 IEEE Winter Conf. on Applicat. of Comput. Vis. (WACV), Lake Tahoe, NV, 2018, pp. 1451-1460.

[21] H. Zhao, J. Shi, X. Qi, X. Wang and J. Jia, "Pyramid Scene Parsing Network," 2017 IEEE Conf. on Comput. Vis. and Pattern Recognit. (CVPR), Honolulu, HI, 2017, pp. 6230-6239.

[22] Ziteng Gao, Limin Wang, and Gangshan Wu. "LIP: Local Importancebased Pooling.” In Proc. ICCV, Oct. 2019, pp. 3355-3364.

[23] V. Badrinarayanan, A. Kendall, and R. Cipolla, "Segnet: A deep convolutional encoder-decoder architecture for image segmentation," in IEEE Trans. on Pattern Anal. and Mach. Intell., vol. 39, no. 12, pp. 2481-2495, Dec 2017.

[24] A. Paszke, A. Chaurasia, S. Kim, and E. Culurciello. (Jun. 2016). "ENet: A deep neural network architecture for real-time semantic segmentation." [Online]. Available: https://arxiv.org/abs/1606.02147

[25] G. Lin, A. Milan, C. Shen and I. Reid, "RefineNet: Multi-path Refinement Networks for High-Resolution Semantic Segmentation," 2017 IEEE Conf. on Comput. Vis. and Pattern Recognit. (CVPR), Honolulu, HI, 2017, pp. 5168-5177.
[26] M. Win, A. R. Bushroa, M. A. Hassan, N. M. Hilman and A. IdeEktessabi, "A Contrast Adjustment Thresholding Method for Surface Defect Detection Based on Mesoscopy," in IEEE Trans. on Ind. Informat., vol. 11, no. 3, pp. 642-649, June 2015.

[27] M. Quintana, J. Torres and J. M. Menéndez, "A Simplified Computer Vision System for Road Surface Inspection and Maintenance," in IEEE Trans. on Intell. Transp. Syst., vol. 17, no. 3, pp. 608-619, March 2016.

[28] X. Bai, Y. Fang, W. Lin, L. Wang and B. Ju, "Saliency-Based Defect Detection in Industrial Images by Using Phase Spectrum," in IEEE Trans. on Ind. Informat., vol. 10, no. 4, pp. 2135-2145, Nov. 2014.

[29] X. Xie and M. Mirmehdi, "TEXEMS: Texture Exemplars for Defect Detection on Random Textured Surfaces," in IEEE Trans. on Pattern Anal. and Mach. Intell., vol. 29, no. 8, pp. 1454-1464, Aug. 2007.

[30] J. Masci, U. Meier, G. Fricout and J. Schmidhuber, "Multi-scale pyramidal pooling network for generic steel defect classification,"

, Dallas, TX, 2013, pp.

$1-8$.

[31] V. Natarajan, T. Hung, S. Vaikundam and L. Chia, "Convolutional networks for voting-based anomaly classification in metal surface inspection," 2017 IEEE Int. Conf. on Ind. Technol. (ICIT), Toronto, ON, 2017, pp. 986-991.

[32] Y. He, K. Song, H. Dong and Y. Yan, "Semi-supervised defect classification of steel surface based on multi-training and generative adversarial network." Opt. and Lasers in Eng., 122 (2019): 294-302.

[33] Y. He, K. Song, Q. Meng and Y. Yan, "An End-to-end Steel Surface Defect Detection Approach via Fusing Multiple Hierarchical Features," in .DOI: 10.1109/TIM.2019.2915404

[34] F. Chen and M. R. Jahanshahi, "NB-CNN: Deep Learning-Based Crack Detection Using Convolutional Neural Network and Naïve Bayes Data Fusion," in IEEE Trans. on Ind. Electron., vol. 65, no. 5, pp. 4392-4400, May 2018.

[35] J. Zhong, Z. Liu, Z. Han, Y. Han and W. Zhang, "A CNN-Based Defect Inspection Method for Catenary Split Pins in High-Speed Railway," in IEEE Trans. on Instrum. and Meas., vol. 68, no. 8, pp. 2849-2860, Aug. 2019.

[36] F. Yang, L. Zhang, S. Yu, D. Prokhorov, X. Mei and H. Ling, "Feature Pyramid and Hierarchical Boosting Network for Pavement Crack Detection," in IEEE Trans. on Intell. Transp. Syst. DOI: 10.1109/TITS.2019.2910595

[37] R. Ren, T. Hung and K. C. Tan, "A Generic Deep-Learning-Based Approach for Automated Surface Inspection," in IEEE Trans. on Cybern., vol. 48, no. 3, pp. 929-940, March 2018.

[38] H. Yang, Y. Chen, K. Song and Z. Yin, "Multiscale Feature-ClusteringBased Fully Convolutional Autoencoder for Fast Accurate Visual Inspection of Texture Surface Defects," in IEEE Trans. on Autom. Sci. and Eng., vol. 16, no. 3, pp. 1450-1467, July 2019.

[39] K. Simonyan A. Zisserman "Very deep convolutional networks for largescale image recognition," in Proc. Int. Conf. Learn. Represent., 2015.

[40] A. Krizhevsky I. Sutskever G. E. Hinton "ImageNet classification with deep convolutional neural networks" ., pp. 1097-1105 2012.

[41] B. Zhou, A. Khosla, A. Lapedriza, A. Oliva, and A. Torralba, "Object detectors emerge in deep scene CNNs," arXiv:1412.6856, 2014.

[42] P. Dollár, R. Appel, S. Belongie and P. Perona, "Fast Feature Pyramids for Object Detection," in IEEE Trans. on Pattern Anal. and Mach. Intell., vol. 36, no. 8, pp. 1532-1545, Aug. 2014.

[43] Wenguan Wang, Shuyang Zhao, Jianbing Shen, Steven C. H. Hoi and Ali Borji, "Salient Object Detection with Pyramid Attention and Salient Edges," 2019 IEEE Conf. on Comput. Vis. and Pattern Recognit. (CVPR), 2019, pp. 1448-1457.

[44] O. Ronneberger, P. Fischer, and T. Brox, "U-net: Convolutional networks for biomedical image segmentation," in Medical Image Computing and Computer-Assisted Intervention MICCAI 2015. Springer International Publishing, 2015, pp. 234-241.

[45] C. Peng, X. Zhang, G. Yu, G. Luo and J. Sun, "Large Kernel Matters Improve Semantic Segmentation by Global Convolutional 
Network," 2017 IEEE Conf. on Comput. Vis. and Pattern Recognit. (CVPR), Honolulu, HI, 2017, pp. 1743-1751.

[46] M. Abadi, P. Barham, and J. Chen, et al., "Tensorflow: A system for large-scale machine learning," May 2016. [Online]. Available: arXiv: 1605.08695 .

[47] DAGM 2007 Datasets. [Online]. Available: https://hci.iwr.uniheidelberg.de/node/3616 (accessed on 27 February 2018).

[48] Y. Huang, C. Qiu, and K. Yuan, "Surface defect saliency of magnetic tile," The Visual Computer, pp. 1-12, 2018.

[49] F. Yu, D. Wang, E. Shelhamer and T. Darrell, "Deep Layer Aggregation," 2018 IEEE/CVF Conf. on Comput. Vis. and Pattern Recognit., Salt Lake City, UT, 2018, pp. 2403-2412

[50] Q. Hou, M. Cheng, X. Hu, A. Borji, Z. Tu and P. H. S. Torr, "Deeply Supervised Salient Object Detection with Short Connections," in IEEE Trans. on Pattern Anal. and Mach. Intell., vol. 41, no. 4, pp. 815-828, 1 April 2019.

[51] K. Song and Y. Yan, "A noise robust method based on completed local binary patterns for hot-rolled steel strip surface defects," Appl. Surface Sci., vol. 285, pp. 858-864, Nov. 2013.

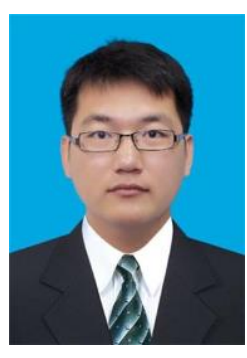

Hongwen Dong received the B.S. degree in School of Mechanical Engineering and Automation, Liaoning University of Technology, Jinzhou, China, in 2016, and the M.S. degree in School of Mechanical Engineering and Automation, Northeastern University, Shenyang, China, in 2018. He is currently pursuing the Ph.D. degree with School of Mechanical Engineering and Automation, Northeastern University, China. His research interests include deep learning, pattern recognition and semantic segmentation.

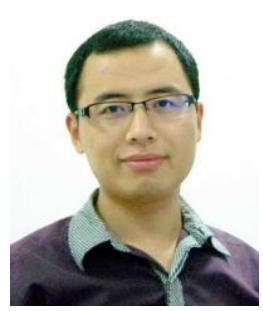

Kechen Song received the B.S., M.S. and Ph.D. degrees in School of Mechanical Engineering and Automation, Northeastern University, Shenyang, China, in 2009, 2011 and 2014, respectively. Between 2018 and 2019, he was an Academic Visitor in the Department of Computer Science, Loughborough University, UK. He is currently an Associate Professor in the School of Mechanical Engineering and Automation, Northeastern University. His research interest covers vision-based inspection system for steel surface defects, surface topography, image processing and pattern recognition.

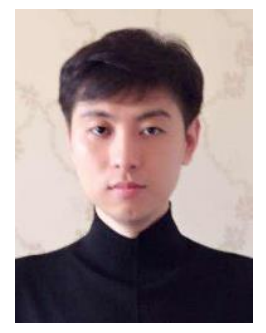

Yu He received the B.S. degree in School of Mechanical Engineering and Automation, Liaoning Technical University, Fuxin, China, in 2014, and the M.S. degree in School of Mechanical Engineering and Automation, Northeastern University, Shenyang, China, in 2016. He is currently pursuing the Ph.D. degree with School of Mechanical Engineering and Automation, Northeastern
University, China. His research interests include deep learning, pattern recognition and intelligent inspection.

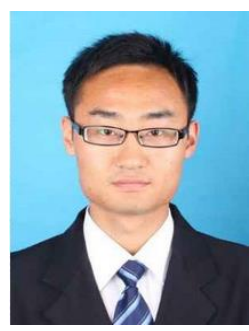

Jing Xu received his B.S., M.S. degrees in School of Mechanical Engineering, Liaoning Shihua University, Fushun, China, in 2013 and 2016, respectively. He is currently pursuing the $\mathrm{PhD}$ degree with School of Mechanical Engineering and Automation, Northeastern University, Shenyang, China. His research interests include robot motion planning and robot control.

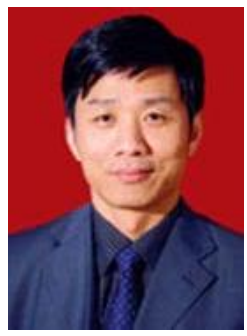

Yunhui Yan received the B.S., M.S. and Ph.D. degrees in School of Mechanical Engineering and Automation, Northeastern University, Shenyang, China, in 1981, 1985 and 1997, respectively. He has been a teacher in Northeastern University of China since 1982, and became as professor in 1997. During 1993-1994, he stayed in the Tohoku National Industrial Research Institute as a visiting scholar. His research interest covers intelligent inspection, image processing and pattern recognition.

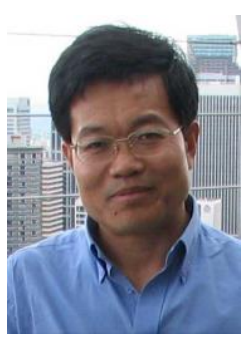

Qinggang Meng (M'06-SM'18)received the B.S. and M.S. degrees from the School of Electronic Information Engineering, Tianjin University, China, and the Ph.D. degree in computer science from Aberystwyth University, U.K. He is a Professor with the Department of Computer Science, Loughborough University, U.K. His research interests include biologically and psychologically inspired learning algorithms and developmental robotics, service robotics, robot learning and adaptation, multi-UAV cooperation, drivers distraction detection, human motion analysis and activity recognition, activity pattern detection, pattern recognition, artificial intelligence, and computer vision. He is a fellow of the Higher Education Academy, U.K. 